\title{
BMJ Open Cognitive effects of non-surgical brain stimulation for major depressive disorder: protocol for a systematic review and meta-analysis
}

\author{
Maximilian Kiebs, ${ }^{1}$ René Hurlemann, ${ }^{1}$ Julian Mutz ${ }^{2,3}$
}

To cite: Kiebs M, Hurlemann R, Mutz J. Cognitive effects of non-surgical brain stimulation for major depressive disorder: protocol for a systematic review and meta-analysis. BMJ Open 2019:9:e023796. doi:10.1136/ bmjopen-2018-023796

- Prepublication history and additional material for this paper are available online. To view these files, please visit the journal online (http://dx.doi. org/10.1136/bmjopen-2018023796).

Received 24 April 2018

Revised 28 August 2018

Accepted 5 December 2018

Check for updates

(C) Author(s) (or their employer(s)) 2019. Re-use permitted under CC BY-NC. No commercial re-use. See rights and permissions. Published by BMJ.

${ }^{1}$ Department of Psychiatry and Division of Medical Psychology, University Hospital Bonn, Bonn, Germany

${ }^{2}$ Faculty of Medicine, School of Public Health, Department of Epidemiology and Biostatistics, Imperial College London,

London, UK

${ }^{3}$ Institute of Psychiatry,

Psychology and Neuroscience,

Social Genetic and

Developmental Psychiatry

Centre, King's College London, London, UK

Correspondence to

Mr. Julian Mutz;

julian.mutz@gmail.com

\section{ABSTRACT}

Introduction Non-surgical brain stimulation techniques may be considered as alternative or add-on treatments for patients with major depressive disorder who failed to respond to pharmacological interventions. Electroconvulsive therapy has been shown to be highly effective in reducing depressive symptoms but stakeholders remain concerned about adverse cognitive effects. Repetitive transcranial magnetic stimulation and transcranial direct current stimulation may be associated with more benign adverse effect profiles and may indeed improve certain cognitive functions such as memory and attention. To guide clinical decision-making, we will carry out a systematic review and meta-analysis of the cognitive effects of eight non-surgical brain stimulation techniques. Methods and analysis A systematic literature search of the Embase, PubMed/MEDLINE and PsycINF0 databases, the Cochrane Central Register of Controlled Trials, ClinicalTrials.gov and OpenGrey will be performed. We will include both randomised clinical trials which report on at least one cognitive measure post treatment as well as non-randomised trials and pre-post intervention studies. There are no restrictions to the type of cognitive outcome measures, except that the tests are standardised and psychometrically validated. The Revised Cochrane tool for assessing risk of bias in randomised trials (RoB 2.0) will be used to evaluate included trials. Pre-post studies will be evaluated using the quality assessment tool developed by the US National Heart, Lung and Blood Institute. Metaanalysis, meta-regression, subgroup and sensitivity analyses will be conducted where sufficient data are available.

Ethics and dissemination No ethical approval is needed to conduct this work. The findings will be submitted for publication in peer-reviewed journals and presented at scientific meetings.

PROSPERO registration number CRD42018118850.

\section{INTRODUCTION}

The immense disease burden of major depressive disorder (MDD) ${ }^{1}$ and unsatisfactory response rates to pharmacological and psychological interventions ${ }^{2-4}$ highlight the need to further develop treatment alternatives. Meta-analyses of non-surgical
Strengths and limitations of this study

- This will be the first comprehensive review to systematically evaluate the cognitive effects of a broad range of non-surgical brain stimulation techniques.

- Findings from our review will inform clinical decision-making beyond estimates of antidepressant efficacy.

- Two investigators will independently conduct the literature search, extract data and evaluate included studies.

- Although we will search multiple electronic databases and grey literature, relevant data that have not been reported may be missed.

brain stimulation including electroconvulsive therapy (ECT), repetitive transcranial magnetic stimulation (rTMS) and more recent modalities such as magnetic seizure therapy (MST) and transcranial direct current stimulation (tDCS) provide evidence for at least modest antidepressant efficacy. ${ }^{5-10}$ Both rTMS and tDCS have been extensively used in research to study and modulate cognitive and psychomotor parameters. When applied over the left dorsolateral prefrontal cortex (DLPFC), there is some evidence to suggest that both techniques improve attention, working memory and psychomotor speed in healthy participants. ${ }^{11} 12$ Modulating cognitive functions is also relevant to the field of depression because in addition to affective and vegetative symptoms, cognitive deficits frequently occur in patients with MDD. ${ }^{13-17}$ However, patients and practitioners remain concerned about the potential cognitive impairment associated with brain stimulation, particularly following ECT. ${ }^{18}$

Multiple studies have investigated the effects of non-surgical brain stimulation on cognition in MDD. For example, ECT has been shown to improve processing speed, working memory, anterograde memory 
and other aspects of executive functions ${ }^{19}$; but also to impair memory, particularly in the form of retrograde and anterograde amnesia. ${ }^{5} 182021$ Findings from a recent meta-analysis of randomised controlled trials of prefrontal-rTMS suggest improvements in psychomotor speed, visual scanning and set shifting, ${ }^{22}$ while a previous review found little evidence for improvements in cognition across psychiatric disorders. ${ }^{23}$ rTMS is not typically associated with adverse cognitive effects following treatment. ${ }^{24}$ MST seems to improve certain aspects of memory and executive function and is associated with fewer undesired cognitive effects than ECT. ${ }^{8}{ }^{25}$ One study found tDCS to ameliorate cognitive control in MDD patients ${ }^{26}$ but a recent individual patient data meta-analysis found no evidence for cognitive benefits independent of changes in mood. ${ }^{27}$

To what extent non-surgical brain stimulation affects cognitive processes in patients with MDD has not been compared in a systematic manner across multiple treatment modalities, despite being critical for clinical decision-making. Treatments are not only evaluated in terms of antidepressant efficacy but also with respect to acceptability and tolerability. Therefore, we aim to systematically evaluate studies that have investigated the cognitive effects of eight non-surgical brain stimulation techniques in MDD: ECT, MST, rTMS, deep transcranial magnetic stimulation (dTMS), priming transcranial magnetic stimulation (pTMS), synchronised transcranial magnetic stimulation (sTMS), theta burst stimulation (TBS) and tDCS. For a brief description of these interventions see online supplementary material 1 . Specifically, we will examine post-treatment and pre-post differences in cognitive performance. Because there has been considerable debate about how to accurately measure adverse cognitive effects (eg, retrograde amnesia after $\mathrm{ECT}^{28}$ ), we will also provide a narrative review of the cognitive measures used in the included studies.

\section{METHODS AND ANALYSIS}

\section{Criteria for considering studies for this review}

Types of studies and publications

We will include randomised clinical trials (RCTs) comparing one active treatment with another active treatment or a sham control. Both parallel-group and cross-over designs will be eligible for inclusion. We will also include non-randomised trials and pre-post studies. Conference abstracts, comments, editorials, review articles, meta-analyses and case reports or case series will be excluded. We will also exclude publications that reported duplicate data and non-English language publications.

\section{Types of participants}

Participants will need to be adult patients (age $\geq 18$ years) with Research Diagnositc Criteria (RDC), Diagnostic and Statistical Manual of Mental Disorders (DSM-III, DSM-IV, DSM-IV-TR or DSM-5) or International Classification of Diseases (ICD-9 or ICD-10) diagnosis of MDD. Studies that included patients with primary diagnoses other than MDD will be excluded. We will also exclude studies that recruited patients with a specific subtype of depression only (eg, postpartum depression) or if depression was a secondary diagnosis (eg, Parkinson's disease with depression). Finally, we will exclude any pre-clinical studies.

\section{Types of interventions}

Studies have to include at least one of the following treatments: tDCS, rTMS, TBS, dTMS, sTMS, pTMS, ECT and MST. For rTMS, interventions will be grouped according to coil location and stimulation frequency: high-frequency stimulation of the left DLPFC, high-frequency stimulation of the right DLPFC, low-frequency stimulation of the right DLPFC, low-frequency stimulation of the left DLPFC and bilateral stimulation. TBS interventions will be grouped according to coil location and stimulation protocol: intermittent stimulation of the left DLPFC (iTBS), continuous stimulation of the right DLPFC (cTBS) and bilateral stimulation (blTBS). Finally, ECT interventions will be grouped according to electrode placement: bi-frontal, bitemporal, right unilateral (RUL ECT) and for RUL ECT also according to electrical dosage (high-dose and low-dose to moderate-dose). Studies that examined vagus nerve stimulation (VNS), transcutaneous VNS or related interventions will be excluded from our review because these are not brain stimulation treatments per se. We also exclude any studies in which pharmacological or psychological treatment was co-initiated with brain stimulation or studies with concurrent cognitive testing. Only studies which have applied a treatment of sufficient duration will be considered. For tDCS, rTMS, TBS, dTMS, sTMS, pTMS this means a treatment duration of at least 2 weeks and for ECT and MST at least six treatment sessions. ${ }^{24} 29$

\section{Types of outcome measures}

For RCTs, only studies which report on at least one cognitive measure post treatment will be included. For non-randomised trials and pre-post studies, only those that report on cognitive performance at baseline and post-treatment will be included. There are no restrictions to the type of cognitive outcome measures except that the tests are standardised and psychometrically validated.

\section{Search methods for identification of studies}

A systematic literature search of the Embase, PubMed/ MEDLINE and PsycINFO databases (accessed via Ovid) and the Cochrane Central Register of Controlled Trials will initially be performed for articles published between the first date available and June 3, 2018. Once we have extracted all data, this literature search will be repeated to identify any studies that were published in the meantime. The following search terms will be used: (depress\$) AND (transcranial direct current stimulation OR tDCS OR transcranial magnetic stimulation OR TMS OR theta burst stimulation OR TBS OR electroshock therapy OR electro therapy OR electroconvulsive therapy OR shock therapy OR convulsive therapy OR magnetic seizure 
therapy OR MST OR pTMS OR sTMS OR dTMS) AND (attention OR memory OR orientation OR learn\$ OR concentration OR cognit\$ OR executive function OR visuospatial OR language OR verbal OR psychomotor speed OR inhibition OR neuropsychology OR side effect OR adverse event $O R$ adverse effect), limiting searches to studies in humans and English-language publications. A detailed description of the search strategy for each database can be found in online supplementary material 2. We will also screen the reference lists of included studies and recent systematic reviews or meta-analyses. 519222327 30-37 Finally, we will search OpenGrey (http://www.opengrey. eu) and ClinicalTrials.gov for relevant material.

\section{Data collection and analysis}

Selection of studies

Titles and abstracts will be screened for the inclusion and exclusion criteria. In instances where the title and abstract provide insufficient information to determine eligibility for inclusion, the full-text version of the article will be assessed. Two authors will independently conduct the systematic literature search.

\section{Data extraction and management}

All data will be independently extracted by two authors and recorded in a standardised spreadsheet. Additional fields not listed on the original spreadsheet will need to be agreed on by both authors. Any discrepancies will be resolved through discussions and, if necessary, consultation with a third author.

We will extract relevant statistics of the cognitive outcomes such as means and SD. To avoid potential carryover effects in cross-over trials, only data from the initial phase will be used. Two experienced neuropsychologists will review and subsequently group the cognitive tests into cognitive subdomains. ${ }^{38}$ Discrepancies in domain assignment will be resolved through consultation with a third neuropsychologist. Since the assessment of cognitive effects can differ between objective and subjective measures,${ }^{39}$ trials reporting subjective measures only will be included in a narrative review. ${ }^{40}$

To evaluate the presence of clinical and methodological heterogeneity we will examine patient and study design characteristics across all eligible studies. The following data on potential effect modifiers will be extracted from each study.

\section{Participant characteristics}

Sex ( $n$ male and female); age (mean, SD and range in years); baseline and post-treatment scores on the Hamilton Depression Rating Scale (HDRS), Montgomery-Åsberg Depression Rating Scale and Clinical Global Impression Scale; hospitalisation status (outpatient, inpatient or mixed); whether patients with psychotic symptoms were excluded from the study (yes/no); treatment strategy (monotherapy, augmentation therapy or mixed); whether patients were previously unresponsive to medication (yes/no); years of education; duration of illness (in years).

\section{Intervention characteristics}

For the ECT trials we will extract data on pulse width (in $\mathrm{ms}$ ); stimulation intensity (multiples of seizure threshold); electrode placement; number of treatments per week and number of treatment sessions. If the number of treatment sessions was not fixed, we will extract the number of treatment sessions provided (mean and SD). For the TMS and MST trials we will extract data on type of coil and sham procedure; magnet location; stimulation frequency (in $\mathrm{Hz}$ ) for each site; stimulation intensity (percentage of rMT); total number of pulses delivered; peak magnetic field and number of treatment sessions. Similar data will be extracted for TBS trials, also including data on the treatment protocol (iTBS, cTBS or BL TBS). Finally, for tDCS trials we will extract data on location of the anode and cathode; electrode size (in $\mathrm{cm}^{2}$ ); current intensity (in $\mathrm{mA})$ and density $\left(\mathrm{mA} / \mathrm{cm}^{2}\right)$; session duration; number of treatment sessions; cumulative charge (=currentintensity $\times$ session duration $\times$ number of treatment sessions) and duration of active stimulation in the sham condition (in s).

The following data will be extracted as applicable: $n$ responders, remitters and all-cause drop-outs; $n$ patients discontinuing treatment due to undesired effects; crossover design (yes/no); industry sponsorship (yes/no); diagnostic manual used to diagnose MDD; criteria used to define treatment resistance; period of drug washout (in number of days); HDRS version; criteria used to define response and remission; anaesthetic agent and muscle relaxant and in case of drop-outs how missing data were treated.

Data that cannot be directly retrieved from the original publications will be requested from the authors or searched for in previous systematic reviews and meta-analyses.

\section{Assessment of risk of bias in included studies}

Two authors will independently conduct the assessment of risk of bias. Discrepancies will be resolved by consulting a third author.

The Revised Cochrane tool for assessing risk of bias in randomised trials (RoB 2.0) ${ }^{41}$ will be used to evaluate included studies. The RoB 2.0 has five domains: bias arising from the randomisation process; bias due to deviations from intended interventions; bias due to missing outcome data; bias in measurement of the outcome and bias in selection of the reported results. Each trial will receive a risk of bias judgement of 'low risk of bias', 'some concerns' or 'high risk of bias' for each domain. Overall risk of bias judgements will be derived from domainlevel judgements. If feasible, sensitivity analyses will be conducted in studies with different levels of overall risk of bias.

We will assess the quality of pre-post studies by using the quality assessment tool for before-after (pre-post) studies 
with no control group developed by the US National Heart, Lung and Blood Institute. ${ }^{42}$

\section{Grading the strength of evidence}

To judge the quality of evidence of our review, we will use the BMJ Evidence Centre guidelines for Grading of Recommendations Assessment, Development, and Evaluation. ${ }^{43}$ As recommended by Preferred Reporting Items for Systematic Reviews and Meta-Analyses Protocols, ${ }^{44}$ we will map the quality of evidence along the domains of consistency, risk of bias, directedness, precision as well as publication bias onto four levels of evidence (high, moderate, low or very low).

\section{Data synthesis}

If data are appropriate for quantitative synthesis (ie, sufficiently homogenous with respect to design and outcome measure), a random-effects model will be used for analysis because we assume that the underlying true effect size will vary between studies. For RCTs with more than two groups, treatment arms will be combined if these cannot be included as separate comparisons. Only RCTs which show no significant differences between the active and sham group for the cognitive tasks at baseline will be analysed. We will compute ORs and standardised mean differences with $95 \%$ CIs for categorical and continuous outcomes, respectively. Prior to computing standardised mean differences, we will follow Altman and Bland's ${ }^{45}$ guidance for detecting skewness. If we detect evidence for skewed data, we will contact the study authors to provide details on the distribution of the raw data. Studies reporting descriptive statistics indicative of skewed data (eg, median and IQR) will not be included in any meta-analytic estimates. Meta-regression, subgroup and sensitivity analyses will be conducted where sufficient data are available. For example, we aim to conduct meta-regression analyses to assess whether any changes in cognitive processes are best explained by baseline differences in cognitive functioning, treatment-related changes in depression severity or by the intervention itself. Mean age of participants, sex ratio and treatment duration will be entered as covariates in meta-regression analyses. All analyses will be conducted using the 'meta' package ${ }^{46}$ for RStudio. Any studies for which quantitative synthesis is deemed not appropriate will be included in a narrative review.

Heterogeneity between studies will be assessed with the $Q_{T}$ statistic, which estimates whether the variance of effect sizes is greater than what would be expected due to sampling error. A $\mathrm{p}$ value smaller than 0.10 provides an indication for significant heterogeneity. The $\mathrm{I}^{2}$ statistic will also be computed for each analysis to provide a descriptive measure of inconsistency across the results of individual trials included in our analyses. It provides an indication of what percentage of the observed variance in effect sizes reflects real differences in effect sizes as opposed to sampling error. Higgins et al suggested that
25\%, $50 \%$ and $75 \%$ represent little, moderate and high heterogeneity, respectively. ${ }^{47}$

When sufficient studies are available, contour-enhanced funnel plots ${ }^{48}$ and Egger's tests ${ }^{49}$ will be used to visualise and statistically assess small study effects.

\section{Patient and public involvement}

Patients or members of the public were not involved in this research.

\section{ETHICS AND DISSEMINATION}

The findings will be submitted for publication in peer-reviewed journals and presented at scientific meetings. Important protocol amendments will be described in the online supplementary material of any published report of our findings.

Acknowledgements We thank Mr Stevan Nikolin at the University of New South Wales and Professor Peng Xie at Chongqing Medical University for their helpful comments during peer review. We thank the Imperial Open Access Fund for providing the funding for the open access publishing fees.

Contributors MK and JM conceived the project. MK and JM wrote the paper with input from RH. All authors have read and approved the final version of the protocol and take responsibility for its content. JM is the guarantor.

Funding The authors have not declared a specific grant for this research from any funding agency in the public, commercial or not-for-profit sectors.

Competing interests MK has received a lecture fee from Innomed Medizintechnik in 2017 and 2018.

Patient consent for publication Not required.

Ethics approval No ethical approval is needed to conduct this work.

Provenance and peer review Not commissioned; externally peer reviewed.

Open access This is an open access article distributed in accordance with the Creative Commons Attribution Non Commercial (CC BY-NC 4.0) license, which permits others to distribute, remix, adapt, build upon this work non-commercially, and license their derivative works on different terms, provided the original work is properly cited, appropriate credit is given, any changes made indicated, and the use is non-commercial. See: http://creativecommons.org/licenses/by-nc/4.0/.

\section{REFERENCES}

1. Whiteford HA, Degenhardt L, Rehm J, et al. Global burden of disease attributable to mental and substance use disorders: findings from the Global Burden of Disease Study 2010. The Lancet 2013;382:1575-86.

2. Sinyor M, Schaffer $A$, Levitt $A$. The sequenced treatment alternatives to relieve depression $\left(S T A R^{\star} D\right)$ trial: a review. Can J Psychiatry 2010;55:126-35.

3. Souery D, Papakostas GI, Trivedi MH, et al. Treatment-resistant depression. J Clin Psychiatry 2006;67(Suppl 6):16-22.

4. Cuijpers P, van Straten A, Bohlmeijer E, et al. The effects of psychotherapy for adult depression are overestimated: a metaanalysis of study quality and effect size. Psychol Med 2010;40:211.

5. UK ECT Review Group. Efficacy and safety of electroconvulsive therapy in depressive disorders: a systematic review and metaanalysis. Lancet 2003;361:799-808.

6. Haq AU, Sitzmann AF, Goldman ML, et al. Response of depression to electroconvulsive therapy: a meta-analysis of clinical predictors. $J$ Clin Psychiatry 2015;76:1374-84.

7. Brunoni AR, Moffa AH, Fregni F, et al. Transcranial direct current stimulation for acute major depressive episodes: meta-analysis of individual patient data. Br J Psychiatry 2016;208:522-31.

8. Cretaz E, Brunoni AR, Lafer B. Magnetic seizure therapy for unipolar and bipolar depression: a systematic review. Neural Plast 2015;2015:1-9.

9. Mutz J, Edgcumbe DR, Brunoni AR, et al. Efficacy and acceptability of non-invasive brain stimulation for the treatment of adult unipolar 
and bipolar depression: a systematic review and meta-analysis of randomised sham-controlled trials. Neurosci Biobehav Rev 2018;92:291-303.

10. Mutz J, Vipulananthan V, Carter B, et al. Comparative efficacy and acceptability of non-surgical brain stimulation for the acute treatment of adult major depressive episodes: A systematic review and network meta-analysis of 113 randomised clinical trials. bioRxiv 2018.

11. Miniussi C, Ruzzoli M. Transcranial stimulation and cognition. Handb Clin Neurol 2013;116:739-50.

12. Brunoni AR, Vanderhasselt MA. Working memory improvement with non-invasive brain stimulation of the dorsolateral prefrontal cortex: a systematic review and meta-analysis. Brain Cogn 2014;86:1-9.

13. Burt DB, Zembar MJ, Niederehe G. Depression and memory impairment: a meta-analysis of the association, its pattern, and specificity. Psychol Bull 1995;117:285-305.

14. Veiel HO. A preliminary profile of neuropsychological deficits associated with major depression. J Clin Exp Neuropsychol 1997;19:587-603.

15. Zakzanis KK, Leach L, Kaplan E. On the nature and pattern of neurocognitive function in major depressive disorder. Neuropsychiatry Neuropsychol Behav Neurol 1998;11:111-9.

16. Snyder HR. Major depressive disorder is associated with broad impairments on neuropsychological measures of executive function: a meta-analysis and review. Psychol Bull 2013;139:81-132.

17. Rock PL, Roiser JP, Riedel WJ, et al. Cognitive impairment in depression: a systematic review and meta-analysis. Psychol Med 2014;44:2029-40.

18. Sackeim HA. Autobiographical memory and electroconvulsive therapy: do not throw out the baby. J Ect 2014;30:177-86.

19. Semkovska M, McLoughlin DM. Objective cognitive performance associated with electroconvulsive therapy for depression: a systematic review and meta-analysis. Biol Psychiatry 2010;68:568-77.

20. Payne NA, Prudic J. Electroconvulsive therapy: Part I. A perspective on the evolution and current practice of ECT. J Psychiatr Pract 2009;15:346-68.

21. Lisanby $\mathrm{SH}$, Maddox JH, Prudic J, et al. The effects of electroconvulsive therapy on memory of autobiographical and public events. Arch Gen Psychiatry 2000;57:581-90.

22. Martin DM, McClintock SM, Forster JJ, et al. Cognitive enhancing effects of rTMS administered to the prefrontal cortex in patients with depression: a systematic review and meta-analysis of individual task effects. Depress Anxiety 2017;34:1029-39.

23. Martin DM, McClintock SM, Forster J, et al. Does Therapeutic Repetitive Transcranial Magnetic Stimulation Cause Cognitive Enhancing Effects in Patients with Neuropsychiatric Conditions? A Systematic Review and Meta-Analysis of Randomised Controlled Trials. Neuropsychol Rev 2016;26:295-309.

24. Lefaucheur JP, André-Obadia N, Antal A, et al. Evidence-based guidelines on the therapeutic use of repetitive transcranial magnetic stimulation (rTMS). Clin Neurophysiol 2014;125:2150-206.

25. Kayser S, Bewernick BH, Matusch A, et al. Magnetic seizure therapy in treatment-resistant depression: clinical, neuropsychological and metabolic effects. Psychol Med 2015;45:1073-92.

26. Wolkenstein L, Plewnia C. Amelioration of cognitive control in depression by transcranial direct current stimulation. Biol Psychiatry 2013;73:646-51.

27. Martin DM, Moffa A, Nikolin S, et al. Cognitive effects of transcranial direct current stimulation treatment in patients with major depressive disorder: An individual patient data meta-analysis of randomised, sham-controlled trials. Neurosci Biobehav Rev 2018;90:137-45.

28. Semkovska M, Noone M, Carton M, et al. Measuring consistency of autobiographical memory recall in depression. Psychiatry Res 2012;197:41-8.
29. NICE. Guidance on the use of electroconvulsive therapy. Guidance and guidelines. https://www.nice.org.uk/guidance/ta59/chapter/3The-technology (accessed 17 Aug 2018).

30. Gardner BK, O'Connor DW. A review of the cognitive effects of electroconvulsive therapy in older adults. J Ect 2008;24:68-80.

31. Kumar S, Mulsant BH, Liu AY, et al. Systematic review of cognitive effects of electroconvulsive therapy in late-life depression. Am J Geriatr Psychiatry 2016;24:547-65.

32. Ren J, Li H, Palaniyappan L, et al. Repetitive transcranial magnetic stimulation versus electroconvulsive therapy for major depression: a systematic review and meta-analysis. Prog Neuropsychopharmacol Biol Psychiatry 2014;51:181-9.

33. Dybedal GS, Tanum L, Sundet K, et al. Cognitive side-effects of electroconvulsive therapy in elderly depressed patients. Clin Neuropsychol 2014;28:1071-90.

34. Ingram A, Saling MM, Schweitzer I. Cognitive side effects of brief pulse electroconvulsive therapy: a review. J Ect 2008;24:3-9.

35. Tortella G, Selingardi PM, Moreno ML, et al. Does non-invasive brain stimulation improve cognition in major depressive disorder? A systematic review. CNS Neurol Disord Drug Targets 2014;13:1759-69.

36. Serafini G, Pompili M, Belvederi Murri M, et al. The effects of repetitive transcranial magnetic stimulation on cognitive performance in treatment-resistant depression. A systematic review. Neuropsychobiology 2015;71:125-39.

37. Kedzior KK, Schuchinsky M, Gerkensmeier I, et al. Challenges in comparing the acute cognitive outcomes of high-frequency repetitive transcranial magnetic stimulation (HF-rTMS) vs. electroconvulsive therapy $(E C T)$ in major depression: a systematic review. J Psychiatr Res 2017;91:14-17.

38. Oremus $\mathrm{C}$, Oremus $\mathrm{M}$, McNeely $\mathrm{H}$, et al. Effects of electroconvulsive therapy on cognitive functioning in patients with depression: protocol for a systematic review and meta-analysis. BMJ Open 2015;5:e006966.

39. Berman RM, Prudic J, Brakemeier EL, et al. Subjective evaluation of the therapeutic and cognitive effects of electroconvulsive therapy. Brain Stimul 2008;1:16-26.

40. Rodgers M, Sowden A, Petticrew M, et al. Testing Methodological Guidance on the Conduct of Narrative Synthesis in Systematic Reviews: Effectiveness of Interventions to Promote Smoke Alarm Ownership and Function. Evaluation 2009;15:49-73.

41. Higgins J, Sterne JAC, Savović J, et al. A revised tool for assessing risk of bias in randomized trials. Chandler J, ed. Cochrane Methods: Cochrane Database of Systematic Reviews, 2016.

42. Study Quality Assessment Tools. National Heart, Lung, and Blood Institute (NHLBI). https://www.nhlbi.nih.gov/health-topics/studyquality-assessment-tools (accessed 23 Aug 2018).

43. Schünemann $H J$, Schünemann $A H$, Oxman $A D$, et al. Grading quality of evidence and strength of recommendations for diagnostic tests and strategies. BMJ 2008;336:1106-10.

44. Moher D, Shamseer L, Clarke M, et al. Preferred reporting items for systematic review and meta-analysis protocols (PRISMA-P) 2015 statement. Syst Rev 2015;4:1.

45. Altman DG, Bland JM. Statistics Notes: Detecting skewness from summary information. BMJ 1996;313:1200.

46. Schwarzer G. Meta: an R package for meta-analysis, 2007.

47. Higgins JP, Thompson SG, Deeks JJ, et al. Measuring inconsistency in meta-analyses. BMJ 2003;327:557-60.

48. Peters JL, Sutton AJ, Jones DR, et al. Contour-enhanced metaanalysis funnel plots help distinguish publication bias from other causes of asymmetry. J Clin Epidemiol 2008;61:991-6.

49. Egger M, Davey Smith G, Schneider M, et al. Bias in meta-analysis detected by a simple, graphical test. BMJ 1997;315:629-34. 